\title{
Chiral-Chiral Communication Mechanisms and Modulation of the Helical Sense and the Secondary Structure in PPAs ${ }^{+}$
}

\author{
Manuel Fernández-Míguez, Emilio Quiñoá and Félix Freire * \\ Centro Singular de Investigación en Química Biolóxica e Materiais Moleculares (CIQUS) and Departamento \\ de Química Orgánica, Universidad de Santiago de Compostela, 15782 Santiago de Compostela, Spain; \\ + Presented at the 24th International Electronic Conference on Synthetic Organic Chemistry, 15 November- \\ 15 December 2020; Available online: https://ecsoc-24.sciforum.net/. \\ Published: date
}

\begin{abstract}
Helical Senses are found in important biological macromolecules from living organisms. DNA and proteins are the most representative of the importance of helicity. New and important functions emerge from the helicity control. Different poly(phenylacetylene) copolymers made of chiral monomers are being used for the comprehension of communication effects like Sergeants and Soldiers Effect (SSE), which modulate the helical sense and the secondary structure of PPAs. Copolymers made of two chiral monomers, $(R)-\alpha$-methoxy- $\alpha$-(trifluoromethyl)phenylacetic acid (MTPA) and (S/R)- $\alpha$-methoxy- $\alpha$-phenylacetic acid (MPA) derivatives, can exhibit complex systems in different solvents following classic or novel effects. Under certain conditions, we can find systems that follow lack of communication, Chiral Conflict and Chiral Accord, that will emerge depending on the helical sense of the two chiral monomers. In relation to this control of $P$ and $M$ helix preferences, different applications have been discovered and future upgrades in this field are expected for stationary phases in HPLC, sensors, novel materials, etc.
\end{abstract}

Keywords: helical polymer; copolymer; chiral communication; Chiral Conflict; Sergeants and Soldiers Effect; poly(phenylacetylene)s

\section{Introduction}

Helical polymers have proven, from early stages, efficacy as stationary phases in HPLC when using the static poly(tert-butyl isocyanide)s [1]. Nowadays, dynamic polymers like poly(phenylacetylene)s (PPAs) have also found applications in this field [2].

The main advantage in dynamic polymers is that they respond to external stimuli, for example, adding chiral molecules, changing the solvent or the $\mathrm{pH}$. This incredible behavior opened the door to discover many new effects by Green and coworkers. In 1988, he was able to command the helix [3] and isolate only one helical sense when the different pendants could communicate across the scaffold following the Sergeants and Soldiers Effect (SSE). They also found that the absence of communication followed a pattern which was dependent on the temperature, Chiral Conflict [4].

In our studies we have applied all the Communication effects to PPA copolymers of MPA and MTPA in order to control the helix and isolate homochiral copolymers. Different interactions between pendants appeared for solvents that stabilize opposite conformations for the monomers. 


\section{Methods}

Monomers and polymers were synthesized at inert atmosphere (Argon), using Schlenk lines and anhydrous high-purity solvents.

UV spectra were obtained using a Jasco V-630 spectrophotometer between a wavelength interval of 240 and $500 \mathrm{~nm}$. Each sample was prepared for a concentration of $0.3 \mathrm{mg}$ of polymer $/ \mathrm{mL}$ solvent.

CD spectra were obtained using a Jasco V-630 spectrophotometer between a wavelength interval of 240 and $500 \mathrm{~nm}$. Each sample was prepared for a concentration of $0.3 \mathrm{mg}$ of polymer/mL solvent.

\section{Results and Discussion}

PPA homopolymers made of $(R)$-MTPA $[(R)-2]$ have a pronounced helical sense, stabilizing different conformations in different solvents and consequently, $M$ type or $P$ type helices [5]. This strong capability to induce homochirality makes these pendants suitable to be Sergeants. The possible conformations are cis or trans for the amide group. That being said, non-donor solvents $\left(\mathrm{CHCl}_{3}\right)$ induce trans isomers, (-) CD signal and $M$ type helices. On the other hand, donor solvents (THF) produce an inversion in the helicity towards (+) CD signal and $P$ type helixes due to the cis isomer (Figure 1). Polarity also plays an important role stabilizing: antiperiplanar structures in the $\mathrm{O}=\mathrm{C}-\mathrm{C}$ OMe bonds in less polar solvents like THF; and synperiplanar conformations in the presence of polar solvents like DMF or DMSO (Figure 1).
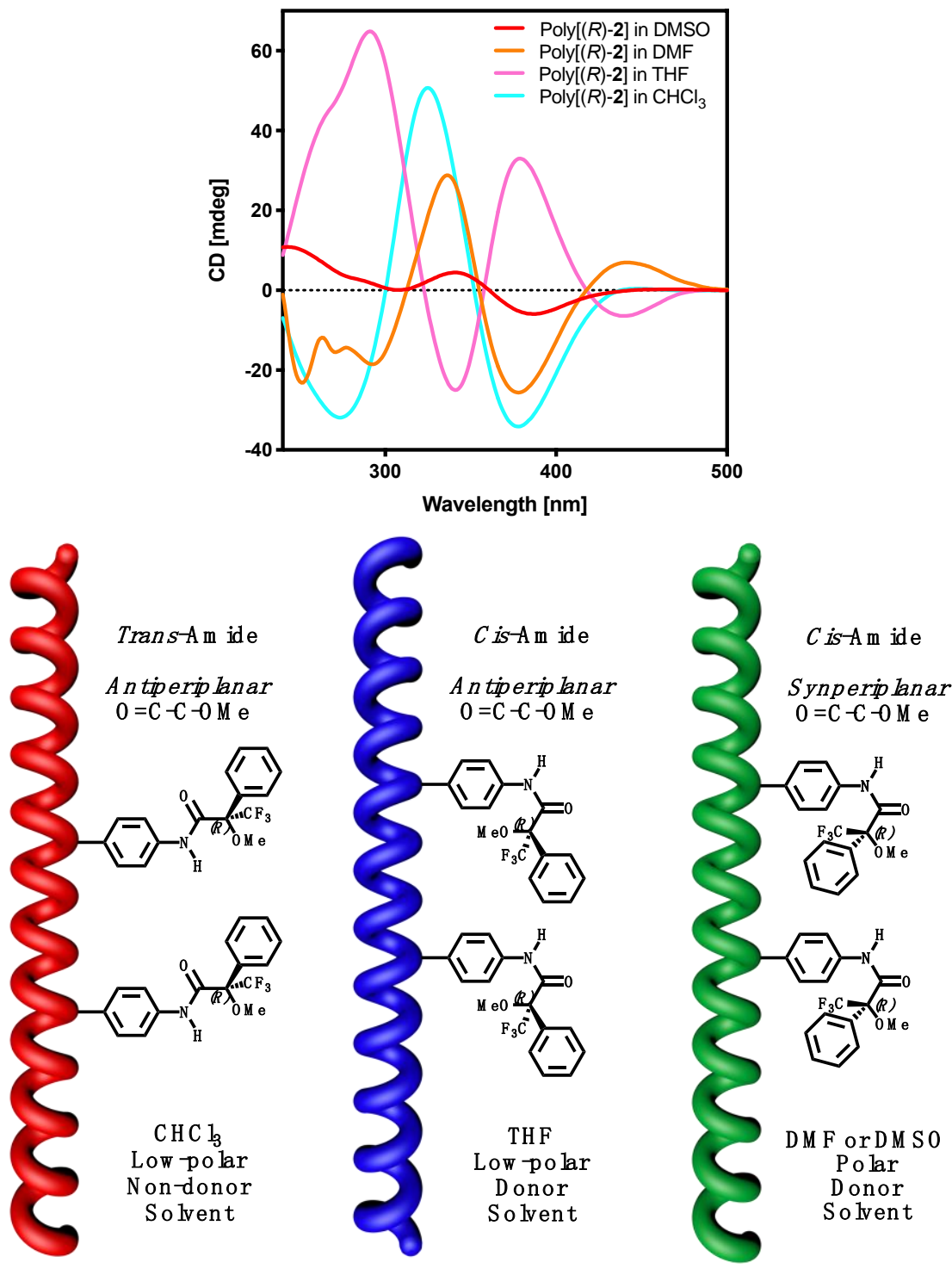
Figure 1. $\mathrm{CD}$ spectra of poly[(R)-2] in DMSO, DMF, THF and $\mathrm{CHCl}_{3}$ solvents. Models for $M$ type or $P$ type helices changing the conformations between cis or trans isomers in the amide group and ap or syn for $\mathrm{O}=\mathrm{C}-\mathrm{C}-\mathrm{OMe}$.

After polymerization, MPA monomers are not able to induce homochirality in the homopolimers because of their racemic conformation. They are called Soldiers as they do not stabilize any of the helical senses. These monomers can be activated to follow the same helical sense of the Sergeants by means of the Sergeants and Soldiers Effect. In contrast, if the Soldiers are commanding the helical sense when activated with low ammounts of Sergeants, the effect will be termed Chiral Coalition [6]. To study these communication effects, copolymers made of the MPA Soldier with the MTPA Sergeant were subjected to solvents with different polarity and donor capability. (R)-MPA and (R)-MTPA homopolymers stabilize opposite helical senses in DMF. The CD signals of the copolymers follow a linear decrease which is a synonymous of a Chiral communication absence. Their scaffolds are different, cis-cisoidal $(c-c)$ isomer at the polyenic backbone for MPA and cistransoidal $(c-t)$ for MTPA, and they stabilize $P$ and $M$ helices (Figure $2 a$ ), respectively. This absence of communication together with the opposite CD signals of the homopolymers are the main points that classify this effect as Chiral Conflict. Analyzing the g value (Figure 2) we can see that we are not at the conflict temperature because $40 \%$ of (R)-2 must have (-) CD signal (Figure 2c). This fenomenum of Chiral Conflict is repeated using the enantiomers in THF (Figure 2d,e).
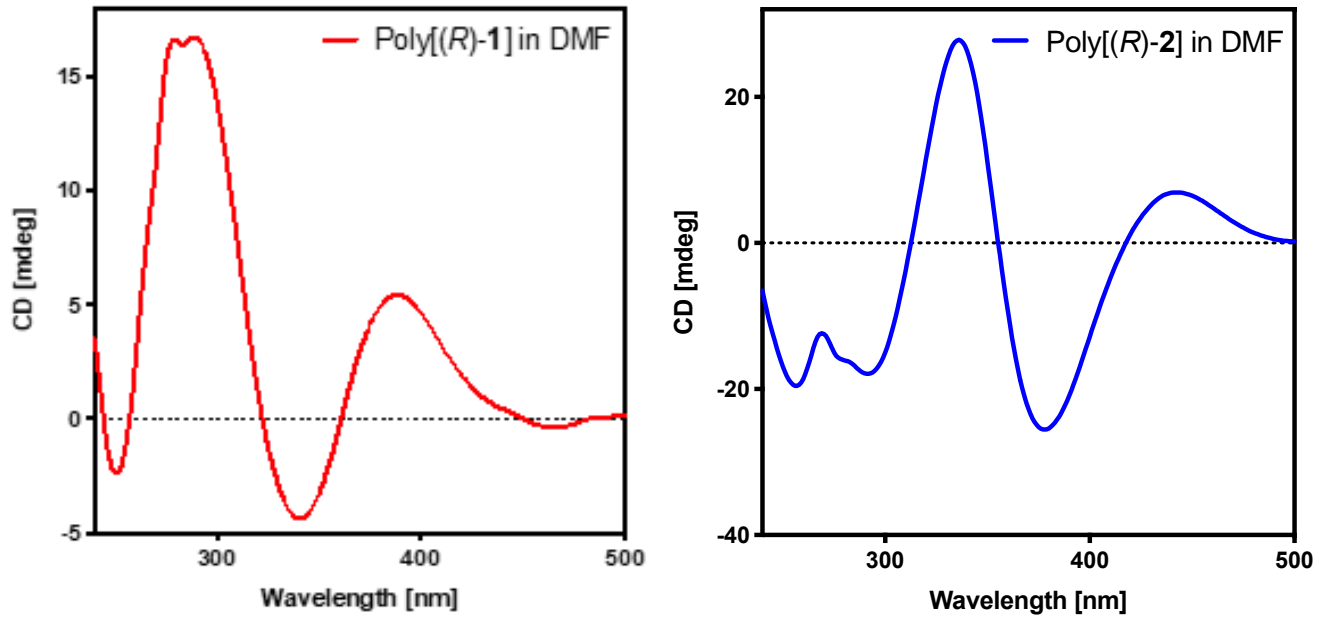

(a)

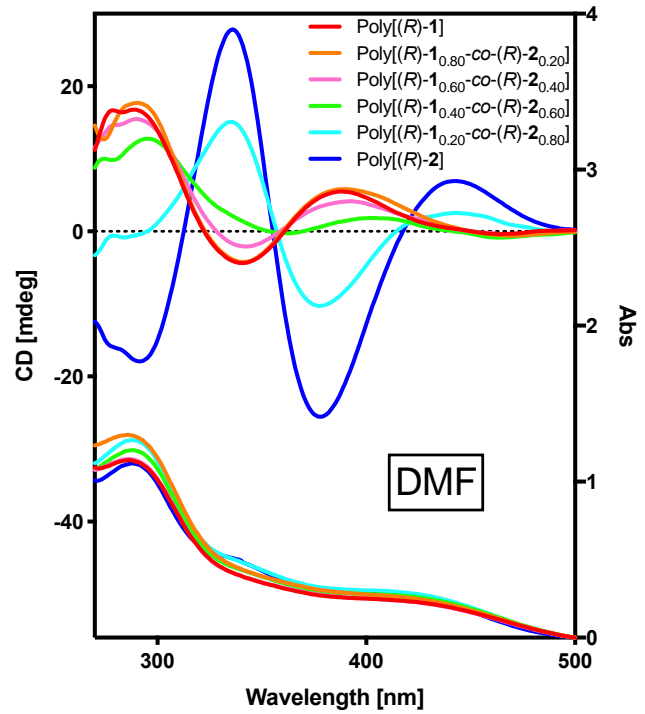

(b) 


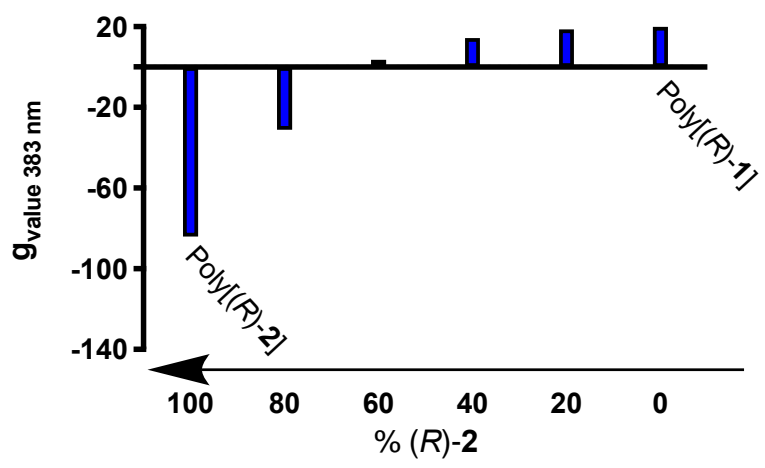

(c)

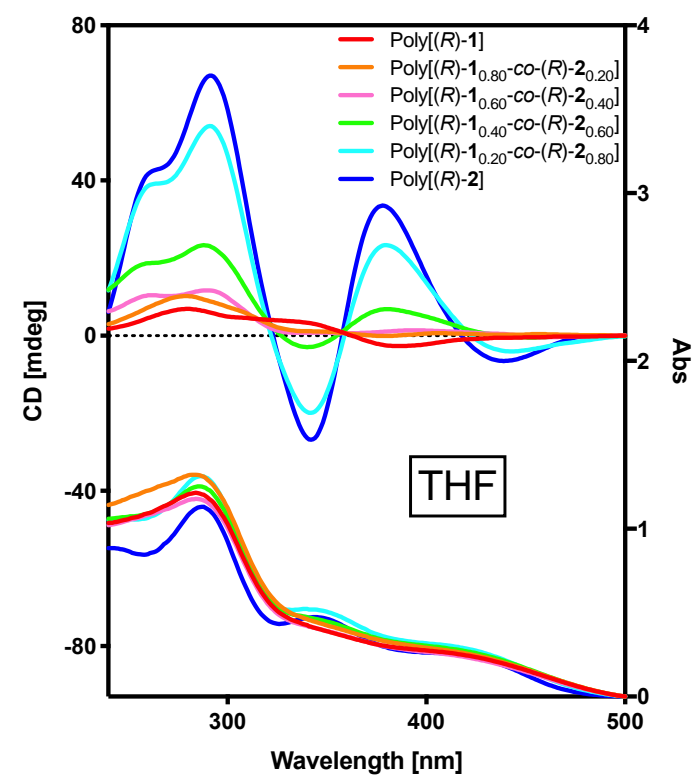

(d)

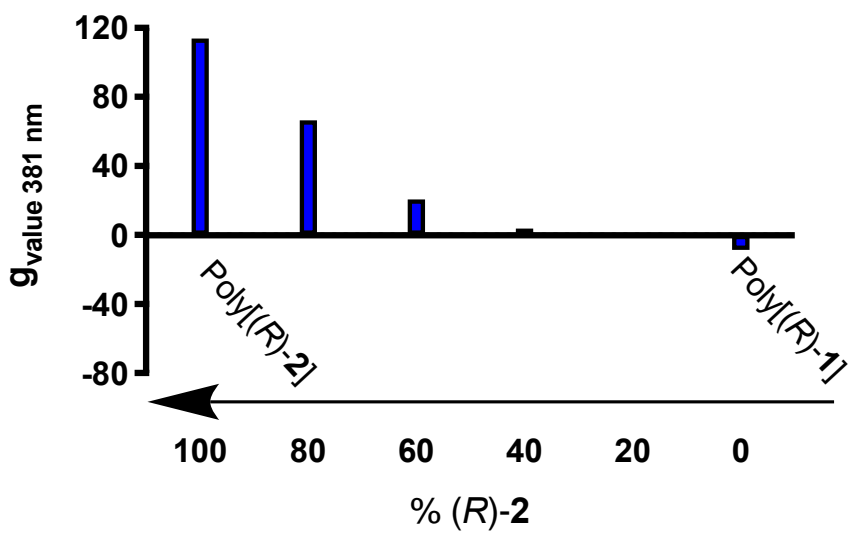

(e)

Figure 2. (a) CD spectra for the homopolymers poly $[(R)-1]$ and poly $[(R)-2]$ with opposite signals in DMF. CD, UV spectra and $g$ values for $(R)-\mathbf{1}$ and $(R)-\mathbf{2}$ copolymers $(\mathbf{b}, \mathbf{c})$ in DMF. $(\mathbf{d}, \mathbf{e})$ in THF.

On the other hand, if we use the (S)-MPA enantiomer in the same solvents (DMF and THF), the helical senses of the homopolymers are inverted. In this case, the two monomers stabilize the same helical sense for the homopolymers poly[(S)-1] and poly[R)-2], anticlockwise in DMF and clockwise 


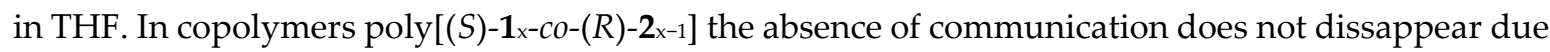
to the same structural difference as before in copolymers poly[(R)-1 $\mathbf{x}-c o-(R)-\mathbf{2} x-1]$. This lack of communication with the stabilization of the same type of helices deffines a system dominated by the Chiral Accord (Figure 3).

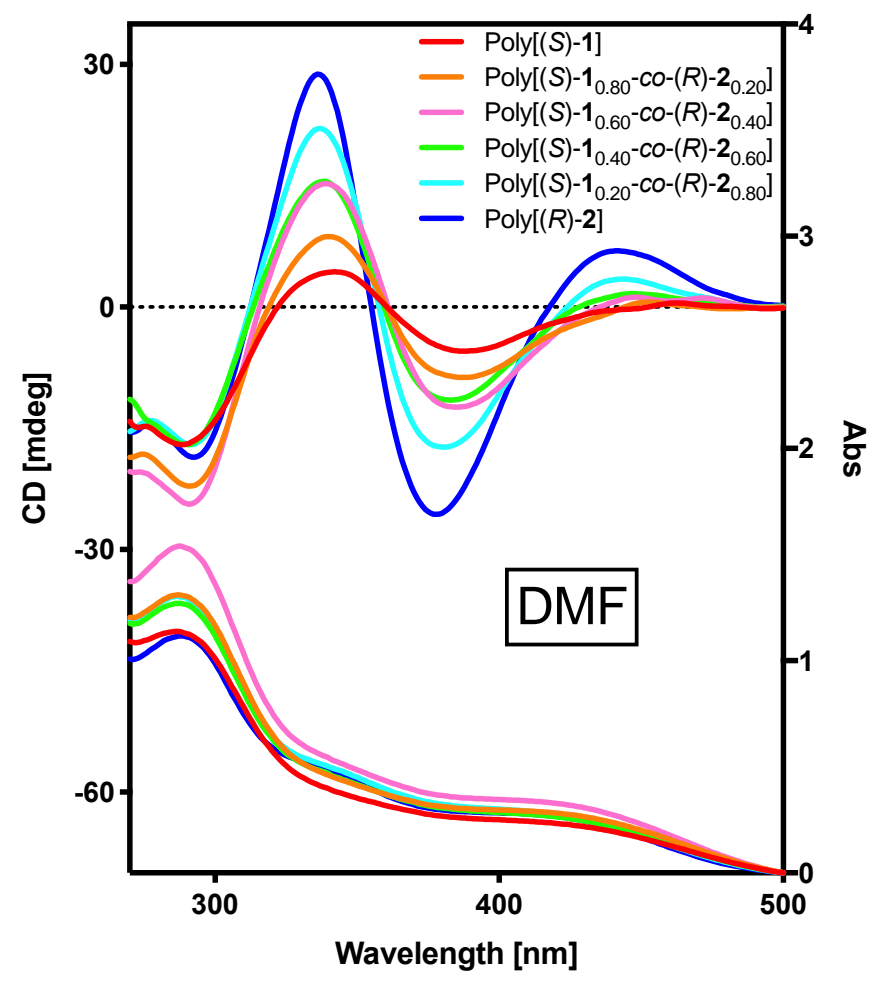

(a)

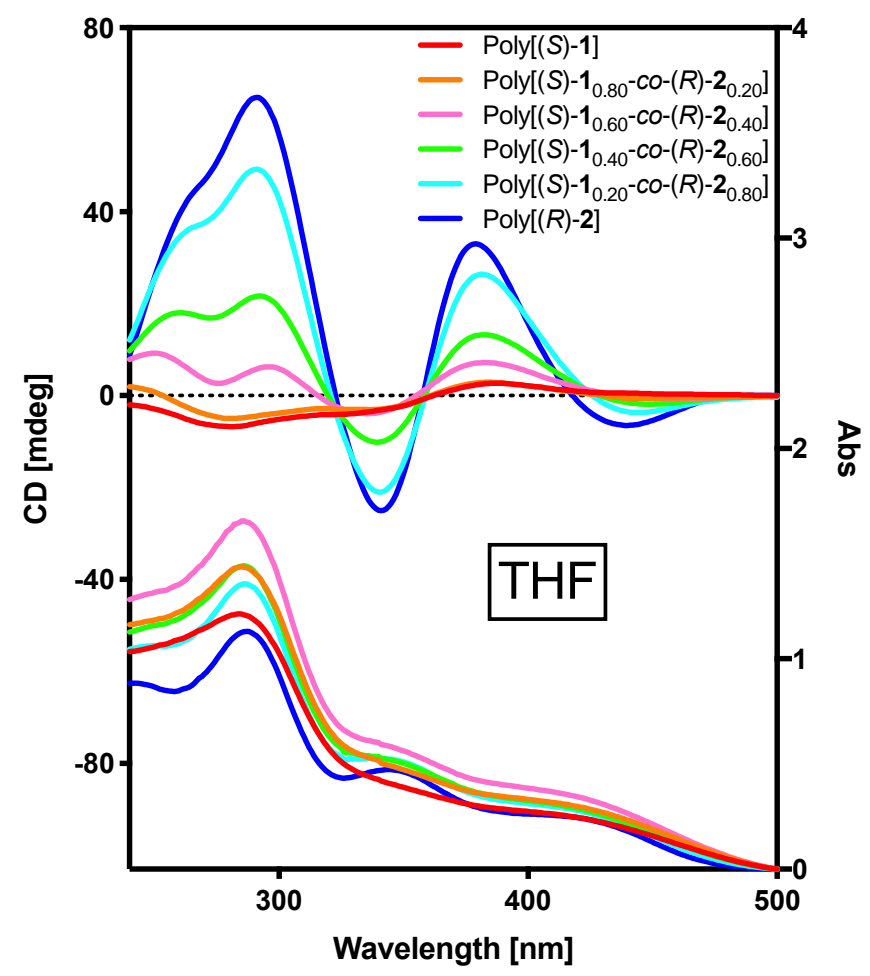

(b) 


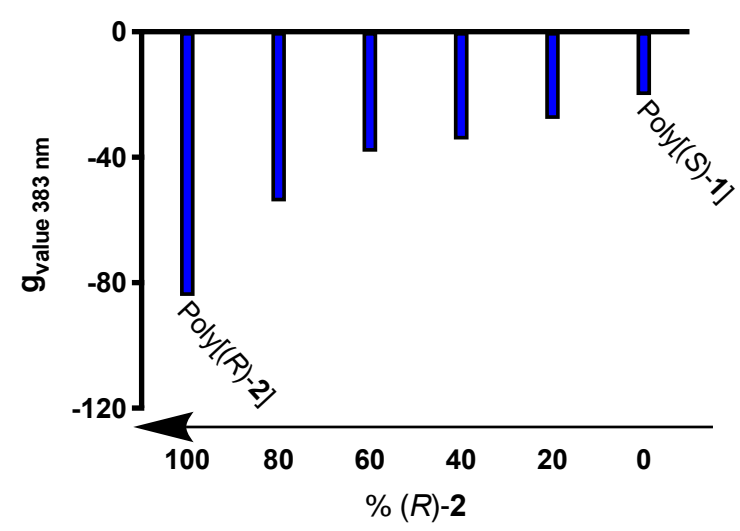

(c)

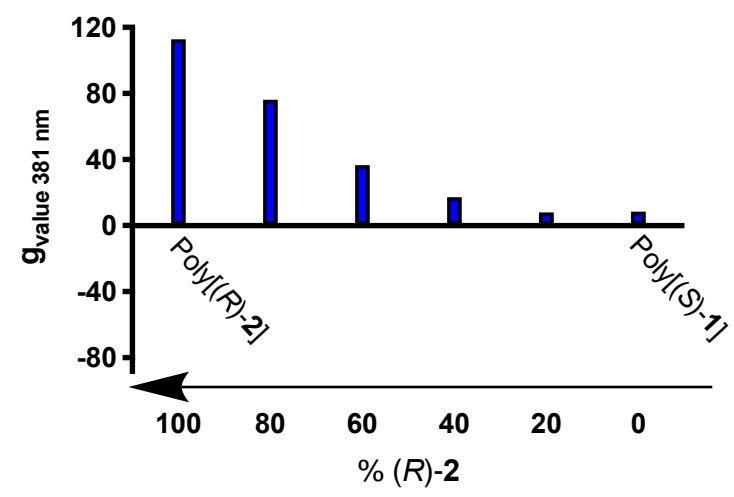

(d)

Figure 3. CD, UV spectra and g values for $(S)-\mathbf{1}$ and $(R)-\mathbf{2}$ copolymers $(\mathbf{a}, \mathbf{b})$ in DMF. (c,d) in THF.

Using $\mathrm{CHCl}_{3}$ as solvent, the scaffolds for the two of the monomers are $c-c$ at the polyenic backbone. Chiral communication effects arise when the two pendants stabilize the same isomer at the inner hellix. In the case of copolymers poly $[(R)-\mathbf{1} x-c o-(R)-\mathbf{2} x-1]$, we can see communication between the pendants due to the non-linear decrease of the CD signal (Figure $4 a, b)$. In copolymers poly[ $\left[(S)-\mathbf{1}_{\mathbf{x}}-\mathrm{Co}-\right.$ $\left.(R)-\mathbf{2}_{\mathrm{x}-1}\right]$ the communication is more percetible with opposite CD signals for poly[(S)-1 $\left.\mathbf{1 0 0}_{00}-\mathrm{co}-(R)-\mathbf{2} 20\right]$ and poly $[(S)-160-C 0-(R)-240]$ to that of the Sergeant homopolymer poly[ $(R)-2]$. This is a clear example of an abnormal Sergeant and Soldiers Effect in which the MTPA Sergeant is inducing a $P$ helix in the (S)-MPA Soldier (Figure 4c,d). 


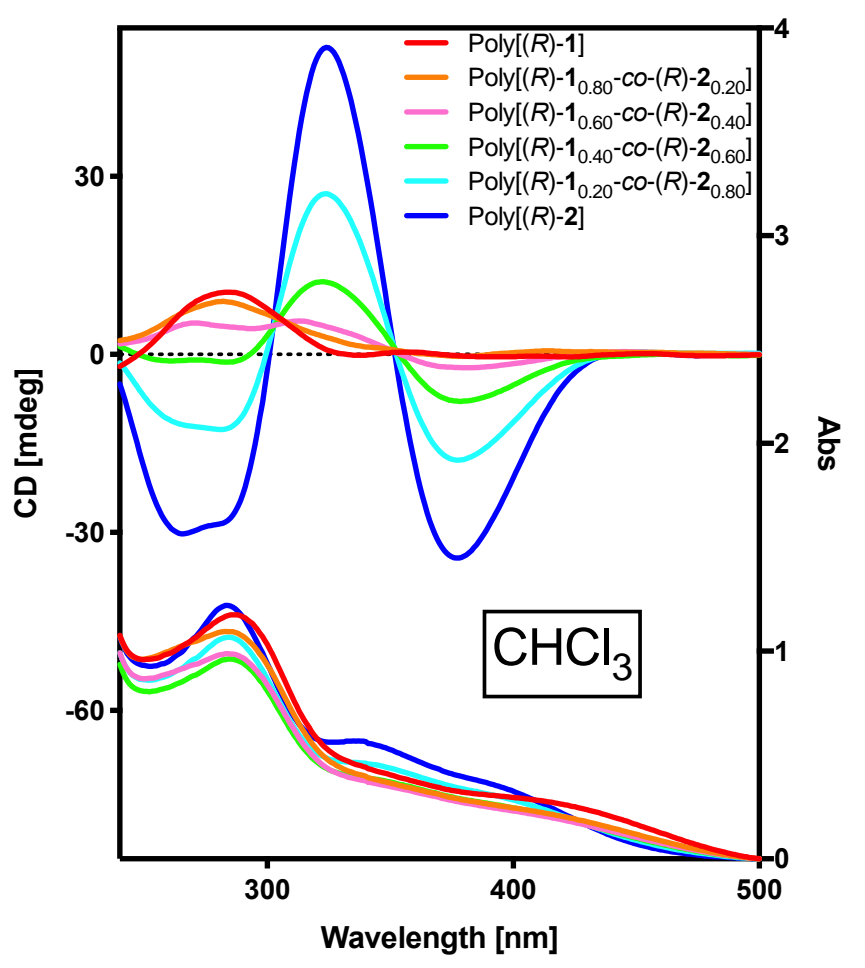

(a)

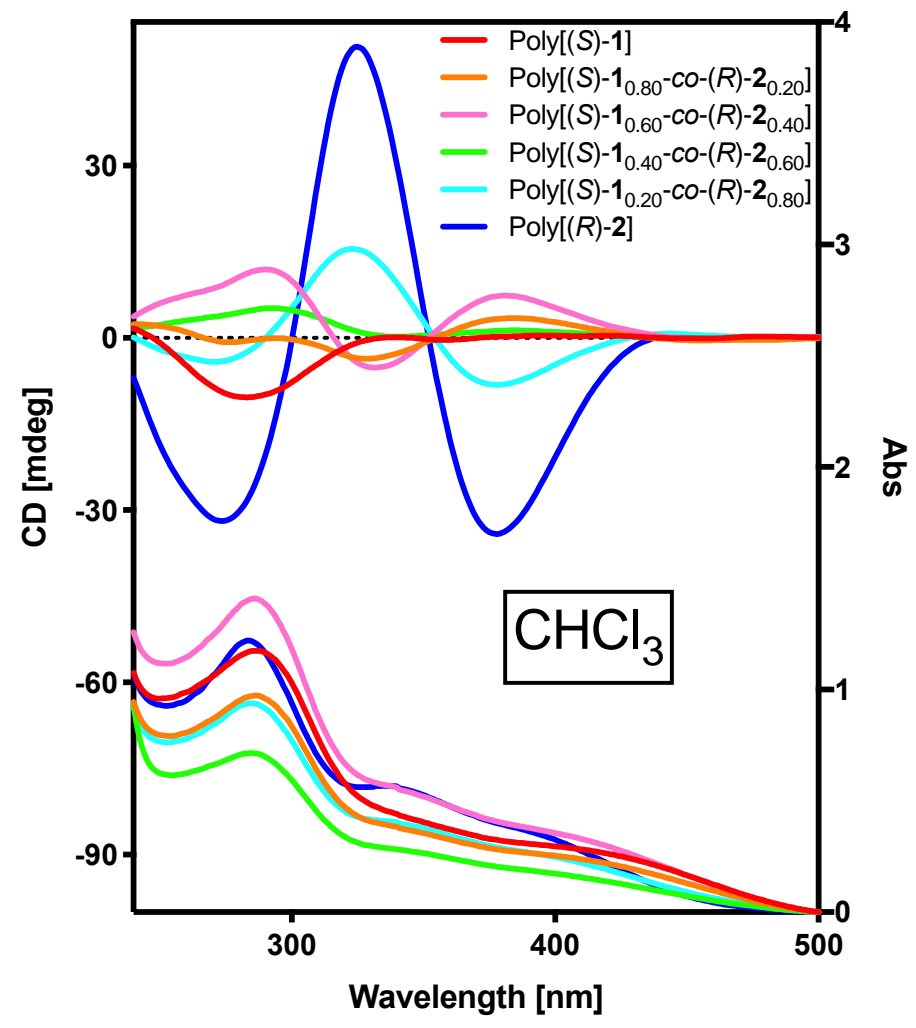

(b) 


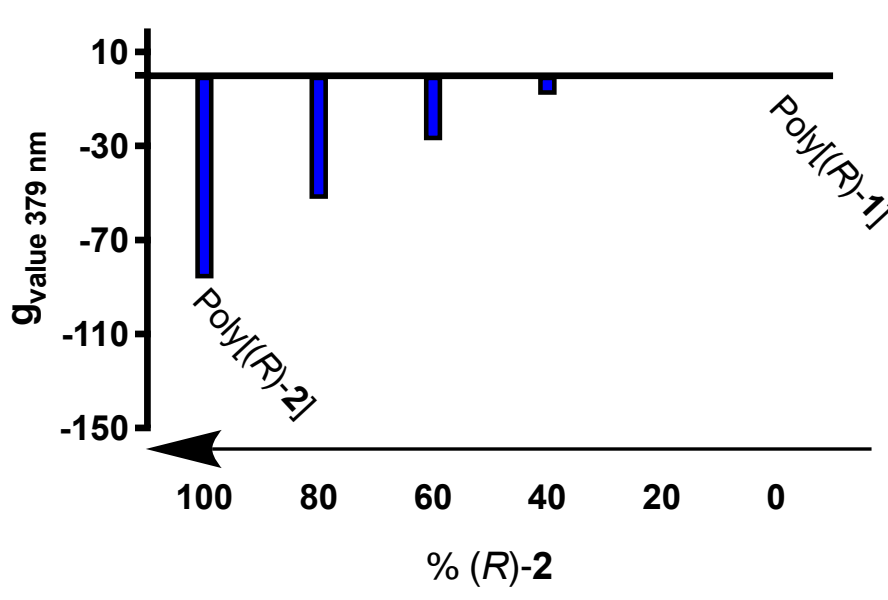

(c)

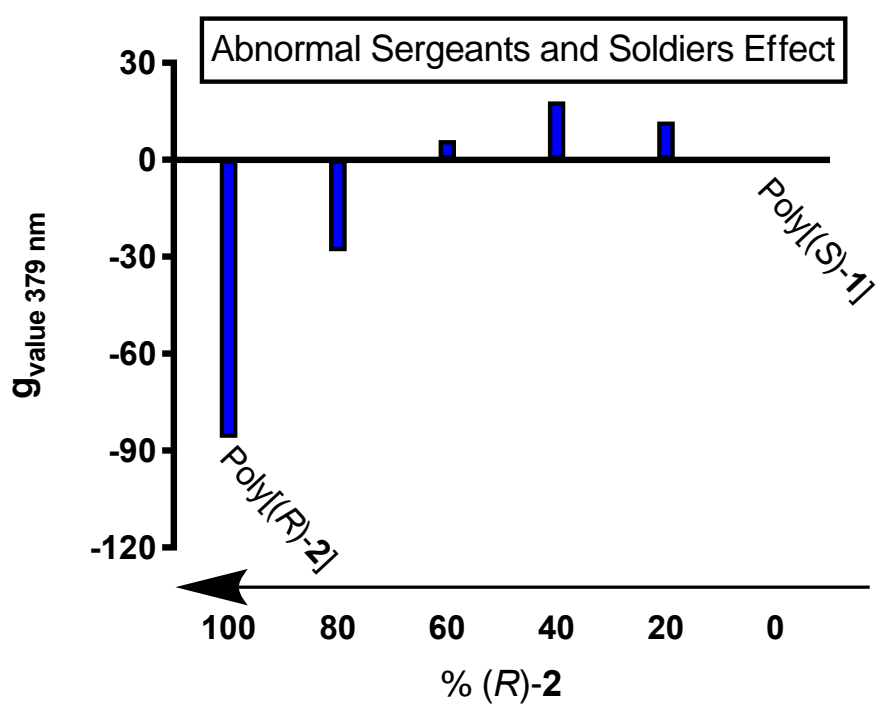

(d)

Figure 3. $\mathrm{CD}$, $\mathrm{UV}$ spectra and $\mathrm{g}$ values in $\mathrm{CHCl}_{3}(\mathbf{a}, \mathbf{b})$ for $(R)-\mathbf{1}$ and $(R)-\mathbf{2}$ copolymers $(\mathbf{c}, \mathbf{d})$ for $(S)-\mathbf{1}$ and $(R)-2$ copolymers.

Despite the fact that DMSO stabilizes $c-t$ scaffolds for MTPA and $c-c$ for MPA, we can find many communication effects in this solvent. The monomers have a prefered helical sense. $(S)-\mathbf{1}$ and $(R)-\mathbf{2}$ stabilize $M$ type helices, while $(R)-\mathbf{1}$ has a prefered $P$ type helix. MPA Soldiers show a bathocromic effect due to the formation of more elongated structures than MTPA Sergeants. When we have an excess of the Soldier in the copolymer, we are in the presence of Chiral Coalition. A low ammount of Sergeant is able to activate the Soldier to stabilize only one helical sense, $(+)$ CD signal and $P$ type helices in $(R)$ configuration and (-) CD signal and $M$ type helices for $(S)$ configuration. They absorbe at same wavelenght $(395 \mathrm{~nm})$.

However, if the ammount of Sergeant in the copolymer is increased, the copolymers will stabilize the poly[(R)-2] type of helix at $388 \mathrm{~nm}$ and will suffer Chiral amplification through Sergeants and Soldiers Effect. The Sergeant commands the helix and stabilize (-) CD signal and $M$ type helices with the two enantiomers of the Soldier. 


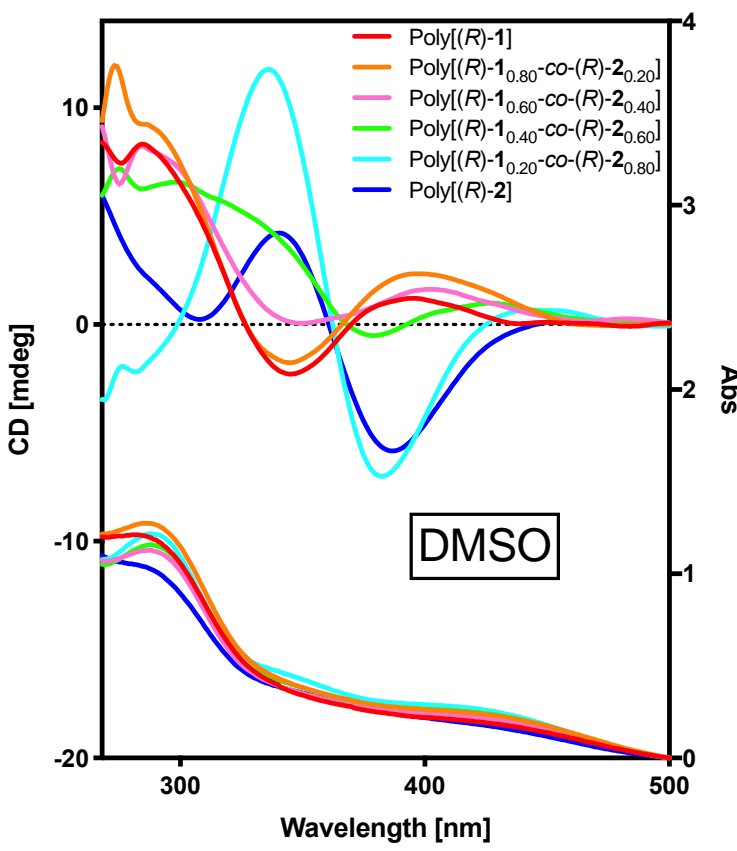

(a)
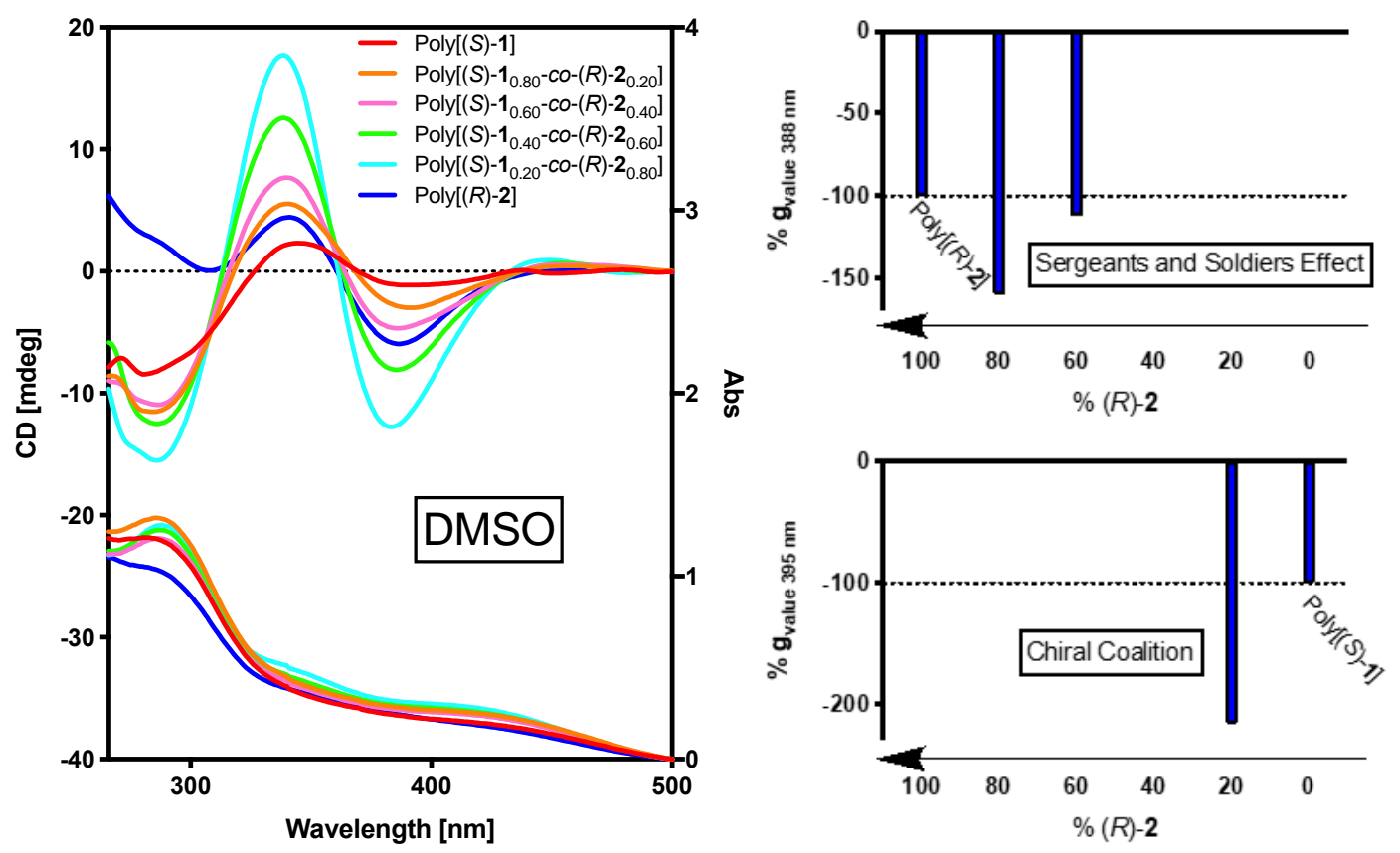

(b)

Figure 4. CD, UV spectra and normalized g values in DMSO (a) $(R)-\mathbf{1}$ and $(R)-\mathbf{2}$ copolymers (b) (S)-1 and $(R)-2$ copolymers.

\section{Conclusions}

To summarize, we have been able to induce chiral communication between the pendants of MPA and MTPA PPAs in DMSO through Sergeants and Soldiers effect and Chiral coalition. In the presence of $\mathrm{CHCl}_{3}$ that stabilized $c-c$ isomers for the two monomers, the helix was commanded by the Sergeant through Abnormal Sergeants and Soldiers effect. In THF and DMF that stabilized different helical structures for MPA $(c-c)$ and MTPA $(c-t)$, copolymers did not show communication following Chiral Conflict or Chiral Accord. 


\section{References}

1. Yuki, H.; Okamoto, Y.; Okamoto, I. Resolution of racemic compounds by optically active poly(triphenylmethyl methacrylate). J. Am. Chem. Soc. 1980, 102, 6356-6358.

2. Hirose, D.; Isobe, A.; Quiñoá, E.; Freire, F.; Maeda, K. Three-State Switchable Chiral Stationary Phase Based on Helicity Control of an Optically Active Poly(phenylacetylene) Derivative by Using Metal Cations in the Solid State. J. Am. Chem. Soc. 2019, 141, 8592-8598.

3. Green, M.M.; Reidy, M.P.; Johnson, R.D.; Darling, G.; O’Leary, D.J.; Willson, G.J. Macromolecular Stereochemistry: The Out-of-Proportion Influence of Optically Active Comonomers on the Conformational Characteristics of Polyisocyanates. The Sergeants and Soldiers Experiment. J. Am. Chem. Soc. 1989, 111, 6452-6454.

4. Tang, K.; Green, M.M.; Cheon, K.S.; Selinger, J.V.; Garetz, B.A. Chiral Conflict. The Effect of Temperature on the Helical Sense of a Polymer Controlled by the Competition between Structurally Different Enantiomers: From Dilute Solution to the Lyotropic Liquid Crystal State. J. Am. Chem. Soc. 2003, 125, 7313-7323.

5. Leiras, S.; Freire, F.; Seco, J.M.; Quiñoá, E.; Riguera, R. Controlled modulation of the helical sense and the elongation of poly(phenylacetylene)s by polar and donor effects. Chem. Sci. 2013, 4, 2735-2743.

6. Arias, S.; Rodríguez, R.; Quiñoá, E.; Riguera, R.; Freire, F. Chiral Coalition in Helical Sense Enhancement of Copolymers: The Role of the Absolute Configuration of Comonomers J. Am. Chem. Soc. 2018, 2, 667-674.

Publisher's Note: MDPI stays neutral with regard to jurisdictional claims in published maps and institutional affiliations.

(C) 2020 by the authors. Submitted for possible open access publication under the terms and conditions of the Creative Commons Attribution (CC BY) license (http://creativecommons.org/licenses/by/4.0/). 